\title{
Periodic Autoregressive Conditional Heteroscedasticity
}

\author{
Tim BOLLERSLEV \\ Department of Economics, Rouss Hall, University of Virginia, Charlottesville, VA 22901, and National Bureau of \\ Economic Research, Cambridge, MA
}

\author{
Eric GHYSELS \\ C.R.D.E., University of Montreal, Montreal, Quebec, $\mathrm{H} 3 \mathrm{C}$ 3J7, Canada, and Centre Interuniversitaire de Recherche \\ en Analyse des Organisations (CIRANO) Montreal, Quebec, Canada
}

\begin{abstract}
Most high-frequency asset returns exhibit seasonal volatility patterns. This article proposes a new class of models featuring periodicity in conditional heteroscedasticity explicitly designed to capture the repetitive seasonal time variation in the second-order moments. This new class of periodic autoregressive conditional heteroscedasticity, or $\mathrm{P}-\mathrm{ARCH}$, models is directly related to the class of periodic autoregressive moving average (ARMA) models for the mean. The implicit relation between periodic generalized ARCH (P-GARCH) structures and time-invariant seasonal weak GARCH processes documents how neglected autoregressive conditional heteroscedastic periodicity may give rise to a loss in forecast efficiency. The importance and magnitude of this informational loss are quantified for a variety of loss functions through the use of Monte Carlo simulation methods. Two empirical examples with daily bilateral Deutschemark/British pound and intraday Deutschemark/U.S. dollar spot exchange rates highlight the practical relevance of the new P-GARCH class of models. Extensions to discrete-time periodic representations of stochastic volatility models subject to time deformation are briefly discussed.
\end{abstract}

KEY WORDS: ARCH; Exchange rates; Periodic structures; P-GARCH; Seasonality; Volatility clustering.

Seasonality in financial-market volatility is pervasive. For instance, Gallant, Rossi, and Tauchen (1992) reported that the historical variance of the Standard and Poor's composite stock-price index in October is almost ten times the variance for March; see also Schwert (1990) and Glosten, Jagannathan, and Runkle (1993). Similarly, Bollerslev and Hodrick (in press) found evidence for significant seasonal patterns in the conditional heteroscedasticity of monthly stock-market dividend yields. At the daily frequency, French and Roll (1986) and Baillie and Bollerslev (1989) demonstrated that daily stock-return and foreignexchange-rate volatility tend to be higher following nontrading days, although proportionally less than during the time period of the market closure. At the intraday level, Wood, McInish, and Ord (1985) documented the existence of a distinct U-shaped pattern in the variances of stock returns over the course of the trading day. Equally pronounced patterns in the volatility of intraday foreign-exchange rates were characterized by Baillie and Bollerslev (1991), Harvey and Huang (1991), and Dacorogna, Müller, Nagler, Olsen, and Pictet (1993).

Although these pronounced daily and intraday seasonal patterns are arguably irrelevant for the analysis of data recorded at lower frequencies, the increased availability of high-frequency financial time series has stimulated a large recent interest in a variety of new modeling issues explicitly related to such data. For instance, several authors have investigated the interrelation between returns in geographically separated financial markets that trade sequentially with little, if any, overlap in their trading hours. The focus of these studies has typically been on the transmission of information as measured by the degree of spillover in the mean returns and/or volatility from one market to the next. Important contributions include those of Engle, Ito, and Lin (1990), who utilized foreign-exchange rates observed at four points during the 24-hour trading day, and Hamao, Masulis, and $\mathrm{Ng}$ (1990), who relied on daily open and close prices for the stock indexes in three countries. A second strand of the intraday time-series-oriented literature has been concerned with the lead-lag relations between two or more markets that trade simultaneously. Examples include Baillie and Bollerslev (1991), who utilized hourly observations on five exchange rates, and Chan, Chan, and Karolyi (1991) who investigated five-minute returns from stock-index and stock-index-futures markets. Finally, a third group of works explored the role of information flow and other microstructure variables as determinants of intraday-return volatility. This literature is exemplified by Bollerslev and Domowitz (1993), who analyzed five-minute foreign-exchange returns, Locke and Sayers (1995), who modeled one-minute stock-index-futures returns, Laux and $\mathrm{Ng}$ (1993) and Foster and Viswanathan (1995), who relied on half-hourly foreign-exchange and equity returns, respectively, and Goodhart, Hall, Henry, and Pesaran (1993), who investigated quote-by-quote returns from the interbank foreign-exchange market. Although many new and interesting results have been uncovered in the just-mentioned studies, on closer inspection the conflicting evidence regarding

(C) 1996 American Statistical Association Journal of Business \& Economic Statistics April 1996, Vol. 14, No. 2 
the volatility dynamics obtained across the different sampling frequencies also raises new important questions concerning the most judicious choice of model structure and the proper treatment of the important intraday seasonal volatility patterns; see Andersen and Bollerslev (in press) for further discussion along these lines.

The purpose of this article is to introduce a new class of parametric time series models for better characterizing the repetitive, or seasonal, patterns in financial-market volatility. To frame the discussion, it is helpful to recall some commonly used time series models for dealing with seasonality in the mean. The framework generally adopted in that context is that of seasonal autoregressive integrated moving average (ARIMA) models, possibly involving an unobserved component structure, as discussed by Nerlove, Grether, and Carvalho (1979), Bell and Hillmer (1984), Hylleberg (1986), and Ghysels (1994), among others. The basic idea of a linear time-invariant autoregressive structure involving seasonal lags can easily be adopted as a possible parameterization for the conditional variance. Such would lead to a seasonal autoregressive conditional heteroscedasticity (ARCH) model, as used for instance by Bollerslev and Hodrick (in press). An alternative approach to analyzing the mean behavior of seasonal time series is to employ ARIMA models whose parameters change seasonally. Initially proposed by Gladyshev (1961), such models have gained considerable interest in recent years. These models, referred to as periodic models because of the seasonal parameter variation, are now well documented both with respect to their theoretical properties and to their empirical relevance. In particular, Tiao and Grupe (1980) established a formal link between the former class of seasonal ARIMA models and periodic ARIMA models; empirical evidence supporting periodic linear structures for a wide variety of macroeconomic time series may be found in the work of Osborn (1988), Osborn and Smith (1989), and Ghysels and Hall (1992), among others.

The same periodic parameter variation originally proposed to capture the repetitive seasonal behavior in the conditional means of economic time series is readily extended to the formulation of conditional heteroscedasticity analogs of periodic ARIMA models. In its simplest form, it is natural to consider a periodic generalized ARCH (GARCH), or P-GARCH, model in which the autoregressive conditional heteroscedasticity is characterized by seasonally varying autoregressive coefficients. In high-frequency financial time series, the repetitive patterns of openings and closures of markets, the number of active markets throughout the day, and so forth are all sources of such periodic variation that must be taken into account. Of course, unlike most cases considered with periodic autoregressive moving average (ARMA) models, the periodic cycles need not be purely repetitive. For instance, the regular nontrading-day cycles associated with weekends is sometimes interrupted by holidays. This necessitates a distinction between purely repetitive cycles and cases in which the periodicity is known but is allowed to vary. For the former case, the results of Tiao and Grupe (1980) for periodic ARMA structures for the mean suggest a similar implicit relationship between the
P-GARCH class of models and weak GARCH-type models with seasonal lags. By this relationship, a seasonal GARCH representation entails an informational loss in efficiency relative to the true P-GARCH model. It is important to recognize, however, that this analog between GARCH models and linear ARMA structures for the mean only goes through for the linear projections figuring in the weak GARCH class of models considered by Drost and Nijman (1993). A strong GARCH structure, which is often implicitly imposed in maximum likelihood estimation, does not yield a direct correspondence between a representation with seasonality in the laws and one with seasonality in the lags. Consequently, the informational loss associated with any neglected periodicity in the parameters may be even more severe for GARCH models than for linear ARMA structures. For the case of a nonrepetitive periodic cycle, somewhat weaker though similar results hold true.

The plan of the rest of the article is as follows. Section 1 is devoted to the definition of the new class of P-GARCH models, along with a brief discussion of their theoretical properties. In Section 2 the actual estimation of P-GARCH models and the finite-sample loss in efficiency from not modeling the periodic autoregressive structure is assessed, through a small-scale Monte Carlo simulation experiment. The estimation results in Section 3 for a daily time series of Deutschemark/British pound exchange rates and an intraday Deutschemark/U.S. dollar exchange-rate series illustrate the practical relevance of periodic ARCH models for characterizing the seasonal volatility patterns in asset returns. Section 4 concludes. Details regarding the mapping between a P-GARCH model and its time-invariant weak GARCH representation are given in the Appendix.

\section{PERIODIC GARCH MODELS}

Following the seminal article by Engle (1982), the ARCH class of models for time-varying conditional heteroscedasticity has become very widely applied. Bollerslev, Chou, and Kroner (1992) provided a survey of empirical applications in finance, and the important theoretical developments were surveyed recently by Bera and Higgins (1993) and Bollerslev, Engle, and Nelson (1994). The $\operatorname{GARCH}(p, q)$ model, introduced by Bollerslev (1986), often provides a parsimonious representation of the volatility dynamics in financial time series. Specifically, this model postulates that the discrete-time real-valued stochastic process $\left\{\varepsilon_{t}\right\}$ satisfies

$$
E\left[\varepsilon_{t} \mid \Omega_{t-1}\right]=0,
$$

and

$$
E\left[\varepsilon_{t}^{2} \mid \Omega_{t-1}\right] \equiv \sigma_{t}^{2}=\omega+\sum_{i=1}^{q} \alpha_{i} \varepsilon_{t-i}^{2}+\sum_{j=1}^{p} \beta_{j} \sigma_{t-j}^{2}
$$

where $\Omega_{t-1}$ denotes the Borel $\sigma$-field filtration based on the realization of the $\left\{\varepsilon_{t}\right\}$ process up to time $t-1$. Equiva- 
lently, Equation (2) may be written as

$$
\varepsilon_{t}^{2}=\omega+\sum_{i=1}^{\max (p, q)}\left(\alpha_{i}+\beta_{i}\right) \varepsilon_{t-i}^{2}+\nu_{t}-\sum_{j=1}^{p} \beta_{j} \nu_{t-j},
$$

where $\nu_{t} \equiv \varepsilon_{t}^{2}-\sigma_{t}^{2}$ and $\alpha_{i} \equiv 0, \beta_{j} \equiv 0$ for $i>q$ and $j>p$, respectively. Note that by definition $E_{t-1}\left(\nu_{t}\right)=0$ so that, if the fourth moment of $\varepsilon_{t}$ is finite, the $\left\{\nu_{t}\right\}$ process is serially uncorrelated. Hence, the representation of the $\operatorname{GARCH}(p, q)$ process in Equation (3) may be interpreted as an ARMA $(\max \{p, q\}, p)$ model for $\left\{\varepsilon_{t}^{2}\right\}$.

Now, instead of having a fixed parameter structure for the conditional-variance equation, it is possible to draw on the similarity of the $\operatorname{ARMA}(p, q)$ model and periodic ARMA processes, and consider a time-varying coefficient model for conditional heteroscedasticity. To define such structures, consider a modified Borel $\sigma$-field filtration in which the usual $\Omega_{t-1}$ is augmented by a process defining the stage of the periodic cycle at each point in time, say $\Omega_{t-1}^{s}$. A more detailed discussion of such $\sigma$ filtrations underlying periodic models for the conditional mean was given by Hansen and Sargent (1990). The class of P-GARCH processes may now be defined as

$$
E\left[\tilde{\varepsilon}_{t} \mid \Omega_{t-1}^{s}\right]=0
$$

and

$$
E\left[\tilde{\varepsilon}_{t}^{2} \mid \Omega_{t-1}^{s}\right] \equiv \tilde{\sigma}_{t}^{2}=\omega_{s(t)}+\sum_{i=1}^{q} \alpha_{i s(t)} \tilde{\varepsilon}_{t-i}^{2}+\sum_{j=1}^{p} \beta_{j s(t)} \tilde{\sigma}_{t-j}^{2},
$$

where $s(t)$ refers to the stage of the periodic cycle at time $t$. Note that $\Omega_{t-1}^{s}$ appears both in the conditional mean and variance equations. Consequently, $\tilde{\varepsilon}_{t}$ may differ from $\varepsilon_{t}$ defined in Equation (1). For instance, $\varepsilon_{t}$ may be the residual from a fixed parameter seasonal ARMA model, whereas $\tilde{\varepsilon}_{t}$ refers to the residuals from a P-ARMA model for the conditional mean. Equation (5) provides the same direct analog of Equation (2) allowing for periodic varying coefficients in the variance equation. Although the lag lengths $p$ and $q$ do not depend on $s(t)$, this entails no loss in generality because $p$ and $q$ may be set to their maximal orders across all stages of the periodic cycle. Of course, for the $\operatorname{P-GARCH}(p, q)$ model to be well defined, the conditional variance, $\tilde{\sigma}_{t}^{2}$, must be positive almost surely. Necessary and sufficient conditions on the $\omega_{s(t)}, \alpha_{i s(t)}$, and $\beta_{j s(t)}$ parameters for this to hold true in general are elusive but may be easily verified on a case-by-case basis following the approach of Nelson and Cao (1992).

The most straightforward P-GARCH model is obtained when the periodic cycle is purely repetitive; that is, $s(t)=t$ modulus $S$, where $S$ is the length of the cycle. An example of such a repetitive cycle would be the intraday pattern in market activity associated with the regular opening and closure of financial markets. In many empirical applications to financial time series, however, $s(t)$ may be governed by a variable predetermined cycle with an upperbound $S$. For example, with daily data nontrading days usually occur every fifth observation, but some weeks have holidays that inter- rupt this regular weekly pattern. In this situation $S=5$, but not all return cycles actually attain five consecutive trading days.

In the existing ARCH literature, the modeling of nontrading-day effects have typically been limited to $\omega_{s(t)}$. The representation in Equation (5), however, allows for a much richer dynamic structure in that the innovations that occur over different time periods may have their own distinct impact on the volatility process through the $\alpha_{i s(t)}$ and $\beta_{j s(t)}$ coefficients. In further interpreting these coefficients, the $\alpha_{i s(t)}$ 's may be viewed as a measure of the immediate, or direct, impact of any news arrivals, but the smooth longterm evolution in the volatility process is captured by the $\beta_{j s(t)}$ coefficients. In many practical applications the periodic variation is therefore naturally constrained to the $\alpha_{i s(t)}$ coefficients, keeping $\beta_{j s(t)} \equiv \beta_{j}$ constant across all stages of the cycle. From Equation (6), this results in a P-GARCH process with periodicity in the autoregressive part of the model only.

Analogous to the ARMA representation for the $\operatorname{GARCH}(p, q)$ model in Equation (3), it is possible to interpret the P-GARCH $(p, q)$ model defined in Equation (5) as a periodic ARMA process for $\left\{\tilde{\varepsilon}_{t}^{2}\right\}$ with a time-varying but periodic correlation structure,

$$
\begin{aligned}
\tilde{\varepsilon}_{t}^{2}=\omega_{s(t)}+\sum_{i=1}^{\max (p, q)}\left(\alpha_{i s(t)}+\beta_{i s(t)}\right) \tilde{\varepsilon}_{t-i}^{2} & \\
& -\sum_{j=1}^{p} \beta_{j s(t)} \tilde{\nu}_{t-j}+\tilde{\nu}_{t},
\end{aligned}
$$

where $\tilde{\nu}_{t} \equiv \tilde{\varepsilon}_{t}^{2}-E\left[\tilde{\varepsilon}_{t}^{2} \mid \Omega_{t-1}^{s}\right] \equiv \tilde{\varepsilon}_{t}^{2}-\tilde{\sigma}_{t}^{2}$. For periodic ARMA models with a purely repetitive cycle of length $S$, Tiao and Grupe (1980) established a characterization of the mapping between the P-ARMA representation and a time-invariant seasonal ARMA model with a cycle of length $S$. Equation (6) suggests the existence of a similar mapping for the P-GARCH class of models with fixed cycles $S$. The similarities between periodic ARMA and periodic GARCH processes do not carry through straightforwardly, however. Because of the nonlinearities, the GARCH class of processes defined in terms of conditional expectations is not closed under temporal and/or cross-sectional aggregation; see Drost and Nijman (1993) and Nijman and Sentana (in press). These difficulties may be circumvented by considering the wider class of weak GARCH processes. In particular, following Drost and Nijman (1993), $\left\{\tilde{\varepsilon}_{t}\right\}$ is defined to follow a weak P-GARCH process when $\tilde{\sigma}_{t}^{2}$ in (5) corresponds to the best linear projection of $\tilde{\varepsilon}_{t}^{2}$ on $\Psi_{t-1}^{s}$, the space spanned by $\left\{1, \tilde{\varepsilon}_{t-1}, \tilde{\varepsilon}_{t-2}, \ldots, \tilde{\varepsilon}_{t-1}^{2}, \tilde{\varepsilon}_{t-2}^{2}, \ldots\right\}$ augmented by $s(t)$; that is,

$$
\begin{aligned}
E\left[\tilde{\varepsilon}_{t}^{2}-\tilde{\sigma}_{t}^{2} \mid \Psi_{t-1}^{s}\right] & =E\left[\left(\tilde{\varepsilon}_{t}^{2}-\tilde{\sigma}_{t}^{2}\right) \tilde{\varepsilon}_{t-i} \mid \Psi_{t-1}^{s}\right] \\
& =E\left[\left(\tilde{\varepsilon}_{t}^{2}-\tilde{\sigma}_{t}^{2}\right) \tilde{\varepsilon}_{t-i}^{2} \mid \Psi_{t-1}^{s}\right]=0,
\end{aligned}
$$

for $i=1,2, \ldots$ The corresponding ARMA representation for the weak P-GARCH model then takes the form

$$
\tilde{\varepsilon}_{t}^{2}=\omega_{s(t)}+\left[\alpha_{s(t)}(L)+\beta_{s(t)}(L)\right] \tilde{\varepsilon}_{t}^{2}+\left[1-\beta_{s(t)}(L)\right] \tilde{\eta}_{t},
$$


with $\alpha_{s(t)}(L) \equiv \Sigma_{i=1 \ldots q} \alpha_{i s(t)} L^{i}, \beta_{s(t)}(L) \equiv \Sigma_{j=1, \ldots, p}$ $\beta_{j s(t)} L^{j}$, and $\tilde{\eta}_{t} \equiv \tilde{\varepsilon}_{t}^{2}-P\left(\tilde{\varepsilon}_{t}^{2} \mid \Psi_{t-1}^{s}\right)$, where $P\left(\cdot \mid \Psi_{t-1}^{s}\right)$ denotes the corresponding linear projection. Note that the projections in (8) still involve seasonal conditioning so that the autocorrelation structure remains periodic. The specification of a weak P-GARCH model obviously entails an informational loss when compared to the P-GARCH formulation in Equation (5), where $\tilde{\sigma}_{t}^{2}$ is defined as the conditional expectation of $\tilde{\varepsilon}_{t}^{2}$ based on the full information set implied by the Borel $\sigma$-field filtration $\Omega_{t-1}^{s}$. By considering the wider class of weak GARCH processes defined previously, however, it is possible to carry out the mechanics of the Tiao and Grupe (1980) formula. This formula essentially amounts to the removal of the seasonal conditioning in (8) by averaging out the autocorrelation structure across all seasons, thus resulting in a time-invariant seasonal weak GARCH process. Further details of this averaging procedure, along with an illustrative example, is provided in the Appendix.

As previously noted, in many financial applications the periodic cycle is not necessarily repetitive with fixed length but rather repetitive with some upperbound $S$. Even though the mechanics of the Tiao and Grupe (1980) formula are no longer directly applicable in this situation, the same basic principle still works. Most importantly, the nonrepetitiveness leads to an average of observations at differently spaced time intervals. A summary discussion of the resulting technical difficulties appears again in the Appendix; for a more thorough discussion of the same issues within the context of time series models for the conditional mean, we refer to Hansen and Sargent (1990).

Of course, the idea of using periodic structures in formulating time series models for conditional heteroscedasticity is not limited to the GARCH class of models. In particular, although the $\operatorname{GARCH}(p, q)$ model in Equation (2) captures the tendency for large absolute returns to be followed by other large absolute returns, the model does not allow for any asymmetric response in the conditional variance function. This may be a reasonable assumption for the two exchange-rate series analyzed in Section 3, but the volatility response function is not symmetric for all financial returns. Notably, stock-market volatility tends to increase less following large positive, compared to negative, return innovations of the same absolute magnitude; for example, see Engle and $\mathrm{Ng}$ (1993). To parsimoniously capture this so-called leverage effect, Nelson (1991) proposed the exponential GARCH, or EGARCH, model. In the EGARCH model $\left\{\ln \left(\sigma_{t}^{2}\right)\right\}$ follows an ARMA process in which positive and negative shocks have their own distinct effect on the future conditional variances. Extending this formulation to allow for seasonal conditioning, the corresponding P-EGARCH $(p, q)$ model becomes apparent:

$$
\begin{aligned}
\ln \left(\tilde{\sigma}_{t}^{2}\right)=\omega_{s(t)}+ & \alpha_{s(t)}(L) \beta_{s(t)}(L)^{-1} \\
& \times\left[\theta_{s(t)} \tilde{z}_{t-1}+\gamma_{s(t)}\left(\left|\tilde{z}_{t-1}\right|-E\left|\tilde{z}_{t-1}\right|\right)\right],
\end{aligned}
$$

where $\tilde{z}_{t} \equiv \tilde{\varepsilon}_{t} \tilde{\sigma}_{t}^{-1}$ has conditional mean 0 and unit variance. Obviously, the process in (9) is easily overparameterized. For instance, with a variable, though perfectly pre- dictable, periodic pattern due to nontrading-day effects only, even the simple P-EGARCH $(1,1)$ model involves a total of 10 parameters. For higher-order models and more complicated periodic cycles, the number of parameters increases rapidly at the rate $S[(p+q)+3]$ so that some simplifying assumptions will have to be imposed. The case in which $\alpha_{s(t)}(L)=\alpha(L)$ and $\beta_{s(t)}(L)=\beta(L)$ for all $s(t)$ corresponds to a process with periodic asymmetries. Hence, a negative shock after, say, a nontrading day may have a different impact than on any other day of the week. Conversely, with $\theta_{s(t)}=\theta$ and $\gamma_{s(t)}=\gamma$ for all $s(t)$, but periodic ARMA polynomials, the seasonal dynamic is similar to the $\mathrm{P}-\operatorname{GARCH}(p, q)$ model in Equation (5). In the remainder of the article, we shall concentrate exclusively on the PGARCH representation.

\section{ESTIMATION AND INFERENCE}

A variety of estimation and testing procedures have been suggested for conducting inference in ARCH-type models; for a more extensive discussion of these procedures, we refer to the survey articles by Bera and Higgins (1993) and Bollerslev et al. (1994). The scope of this section is not to contribute to the basic theory of estimation and hypothesis testing in ARCH models as such. Instead, our aim is merely to comment on some of the specific issues that arise in the estimation and testing of periodic ARCH structures.

To illustrate, let $\theta$ denote the vector of unknown parameters for all $S$ seasons; that is, $\theta \equiv\left(\theta_{1}, \theta_{2}, \ldots, \theta_{s}\right)$, where for the P-GARCH$(p, q)$ model in Equation (5) $\theta_{s} \equiv$ $\left(\omega_{s}, \alpha_{1 s}, \ldots, \alpha_{q s}, \beta_{1 s}, \ldots, \beta_{p s}\right)$ for $s=1, \ldots, S$. The conditional log-likelihood function for a strong P-GARCH model may then be conveniently written as the sum of the corresponding conditional log-likelihoods for each of the $S$ seasonal cycles,

$$
L_{T}\left(\theta \mid \Omega_{0}^{s}\right)=\sum_{t=1}^{T} l_{t}\left(\theta_{s(t)}\right) .
$$

In particular, assuming the one-step-ahead prediction errors to be conditionally normally distributed,

$$
\begin{aligned}
l_{t}\left(\theta_{s(t)}\right)=-.5\left[\ln (2 \pi)+\ln \left(\tilde{\sigma}_{t}\left(\theta_{s(t)}\right)\right)\right. & \\
& \left.+\tilde{\varepsilon}_{t}\left(\theta_{s(t)}\right)^{2} \tilde{\sigma}_{t}^{2}\left(\theta_{s(t)}\right)^{-1}\right] .
\end{aligned}
$$

Under appropriate regularity conditions the maximum likelihood estimates (MLE's) for the true parameters $\theta_{0}$, say $\hat{\theta}_{T}$, obtained by maximizing Equations (10) and (11) then satisfy

$$
T^{1 / 2}\left(\hat{\theta}_{T}-\theta_{0}\right) \rightarrow N\left(0, A\left(\theta_{0}\right)^{-1}\right),
$$

where $A\left(\theta_{0}\right)$ denotes the Hessian evaluated at $\theta_{0}$. In many applications with high-frequency financial data, the assumption of conditional normality underlying Equation (11) may be violated, however. Fortunately, $\hat{\theta}_{T}$ remains consistent under quite general conditions and may be given a quasi MLE (QMLE) interpretation. Because the outer product of the gradients and the inverse of the Hessian do not cancel out in this situation, the asymptotic covariance matrix for the QMLE takes the form $A\left(\theta_{0}\right)^{-1} B\left(\theta_{0}\right) A\left(\theta_{0}\right)^{-1}$, 
where $B\left(\theta_{0}\right)$ denotes the outer product of the gradients; for further discussion along these lines and a formal proof for the $\operatorname{GARCH}(1,1)$ model, see Weiss (1986), Bollerslev and Wooldridge (1992), Lumsdaine (in press), and Lee and Hansen (1994). Moreover, the Monte Carlo evidence of Bollerslev and Wooldridge (1992) and Baillie, Bollerslev, and Mikkelsen (in press) indicates that for moderately large sample sizes the accuracy of the QMLE-based inference procedures with conditionally leptokurtic errors is comparable to that of exact MLE. Even though no formal analytical results are available for the weak GARCH case, the simulations of Drost and Nijman (1992) suggest that for large sample sizes the QMLE procedure is generally very reliable for the estimation of weak GARCH models also.

We now turn to the results from a small-scale simulation study designed to gauge an idea about the reliability of the MLE procedure applied to the estimation of P-GARCH structures. To assess the potential importance of allowing for seasonal conditioning in ARCH modeling, we also report the results for the QMLE of various timeinvariant weak GARCH formulations. The estimations are based on a sample size of $T=2,000$, which is comparable to the number of observations that are available for the daily exchange-rate series analyzed in Section 3.1. The data-generation process (DGP) is a P-GARCH $(1,1)$ model with a periodic cycle of length 2 . The true model parameters are indicated in the note to Table 1. Although $\alpha_{11}+\beta_{1}>1$, it follows from the Appendix that the model is covariance stationarity because $\left(\alpha_{11}+\beta_{1}\right)\left(\alpha_{12}+\beta_{1}\right)<1$. Furthermore, the two unconditional seasonal variances equal $\sigma_{1}^{2}=\left[\omega_{2}\left(\alpha_{11}+\beta_{1}\right)+\omega_{1}\right]\left[1-\left(\alpha_{11}+\beta_{1}\right)\left(\alpha_{12}+\beta_{1}\right)\right]^{-1}=$ 1.1 and $\sigma_{2}^{2}=\left[\omega_{1}\left(\alpha_{12}+\beta_{1}\right)+\omega_{2}\right]\left[1-\left(\alpha_{11}+\beta_{1}\right)\left(\alpha_{12}+\right.\right.$ $\left.\left.\beta_{1}\right)\right]^{-1}=.9$, respectively, whereas the nonseasonal unconditional variance equals unity, $\sigma^{2}=\frac{1}{2}\left(\sigma_{1}^{2}+\sigma_{2}^{2}\right)=1.0$. All of the reported statistics are based on $N=1,000$ replications. To avoid start-up problems, the first 2,000 observations were discarded for each of the replications. Moreover, to start up the recursions for $\tilde{\sigma}_{t}^{2}\left(\theta_{s(t)}\right)$, the initial values for $\tilde{\varepsilon}_{0}^{2}\left(\theta_{s(0)}\right)$ and $\tilde{\sigma}_{0}^{2}\left(\theta_{s(0)}\right)$ were both fixed at their unconditional seasonal sample analog. The normal random variables were generated by the RNDNS subroutine in the GAUSS computer language. Additional simulation results for other P-GARCH and nonperiodic GARCH models with seasonal dummies in the conditional-variance equation are available on request.

It is immediately clear from Panel A of Table 1 that the MLE procedure does a remarkably good job of uncovering the true unknown parameters for the correctly specified P-GARCH $(1,1)$ models in columns 6 and 7. The mean of the parameter estimates, $\bar{\theta}_{T}=N^{-1} \Sigma_{i=1, \ldots, N} \hat{\theta}_{T, i}$, where $\hat{\theta}_{T, i}$ denote the estimate for $\theta_{0}$ from the $i$ th replication, are all very close to their true values. Note that by a central limit theorem argument the Monte Carlo standard error for $\bar{\theta}_{T}$ may be consistently estimated by $N^{-1 / 2} \approx .032$ times the sample standard error across the 1,000 estimates given in parentheses. Note also that these sample standard errors are in close accordance with the average standard-error estimates based on the robustified covariance-matrix estima- tor reported in brackets. Conventional inference procedures for the P-GARCH model based on the asymptotic distribution in (12) with $A\left(\hat{\theta}_{T}\right)^{-1} B\left(\hat{\theta}_{T}\right) A\left(\hat{\theta}_{T}\right)^{-1}$ in the place of $A\left(\theta_{0}\right)^{-1}$ should therefore work well in practice. This is also in accordance with the recent simulation evidence of Lumsdaine (1995) for the MLE estimation of GARCH(1, 1) and $\operatorname{IGARCH}(1,1)$ models that documents good finitesample behavior of the robustified $t$ statistics based on only $T=500$ observations. It may be particularly noteworthy, however, that the Monte Carlo standard errors and the average QMLE standard-error estimates are similarly close for the estimated nonperiodic weak GARCH models. Although no closed-form expression is available for characterizing the true weak GARCH parameters, it is interesting to note that the average estimates for $\alpha_{1}$ approximately equal $\frac{1}{2}\left(\alpha_{11}+\alpha_{12}\right)=.270$ for all three weak GARCH models. Moreover, the corresponding implied unconditional variances based on the average parameter estimates for the three weak GARCH models equal $.966, .982$, and .958 , respectively, whereas the two unconditional seasonal variances for the GARCH model with $\omega_{1} \neq \omega_{2}$ equal .979 and .936 , respectively.

The first two sets of rows in panel B of Table 1 report the average values of the Akaike (1973) information criterion (AIC) and the Schwarz (1978) information criterion (SIC); that is, AIC $=N^{-1} \Sigma_{i=1, \ldots, N} 2 \cdot L_{T}\left(\hat{\theta}_{T, i} \mid \Omega_{0, i}^{s}\right)-2 k$ and SIC $=N^{-1} \Sigma_{i=1, \ldots, N} 2 \cdot L_{T}\left(\hat{\theta}_{T, i} \mid \Omega_{0, i}^{s}\right)-\ln (T) \cdot k$, where $k \equiv \operatorname{dim}\left(\hat{\theta}_{T}\right)$ refers to the number of estimated model parameters. The fractions reported in parentheses give the proportion of times for which a particular model was favored by one of the two information criteria. It is evident that both selection criteria are very effective in discriminating between the true P-GARCH models and the corresponding nonperiodic weak GARCH models. The AIC favors the PGARCH model with $\omega_{1} \neq \omega_{2}$ for $16.7 \%$ of the replications compared to the more parsimonious SIC, which correctly identifies that $\omega_{1}=\omega_{2}$ for $99.3 \%$ of the replications.

The second group of numbers in Panel B reports the average simulated values for various loss functions designed to measure the difference between the true and the estimated conditional variances. The mean squared error criterion $\mathrm{MSE}_{A}=N^{-1} T^{-1} \Sigma_{i=1, N} \Sigma_{t=1, \ldots, T}\left[\tilde{\sigma}_{t, i}^{2}\left(\theta_{0}\right)-\right.$ $\left.\tilde{\sigma}_{t, i}^{2}\left(\hat{\theta}_{T, i}\right)\right]^{2}$ is always minimized for one of the two PGARCH formulations, with a slight advantage for the true DGP that restricts $\omega_{1}=\omega_{2}$. The increase in the value of the $\mathrm{MSE}_{A}$ criterion for the three nonperiodic weak GARCH models is quite dramatic. Although the MSE loss criterion may be a natural choice in evaluating competing estimates for the mean, it is less obvious in a heteroscedasticity environment; see Bollerslev et al. (1994), Lopez (1994), and West, Edison, and Cho (1993) for further discussion along these lines. A natural alternative is to consider the heteroscedasticity-adjusted MSE, or the relative squared error loss, defined by $\mathrm{HMSE}_{A}=$ $N^{-1} T^{-1} \Sigma_{i=1, \ldots, N} \Sigma_{t=1, \ldots, T}\left[\tilde{\sigma}_{t, i}^{2}\left(\theta_{0}\right) \tilde{\sigma}_{t, i}^{2}\left(\hat{\theta}_{T, i}\right)^{-1}-1\right]^{2}$. This criterion favors the P-GARCH model with $\omega_{1}=\omega_{2}$ for $93.0 \%$ of the simulations. The average simulated value for $\mathrm{HMSE}_{A}$ from this model equals only .013, compared 
Table 1. Finite-Sample Distributions for P-GARCH(1, 1) Data-Generating Process

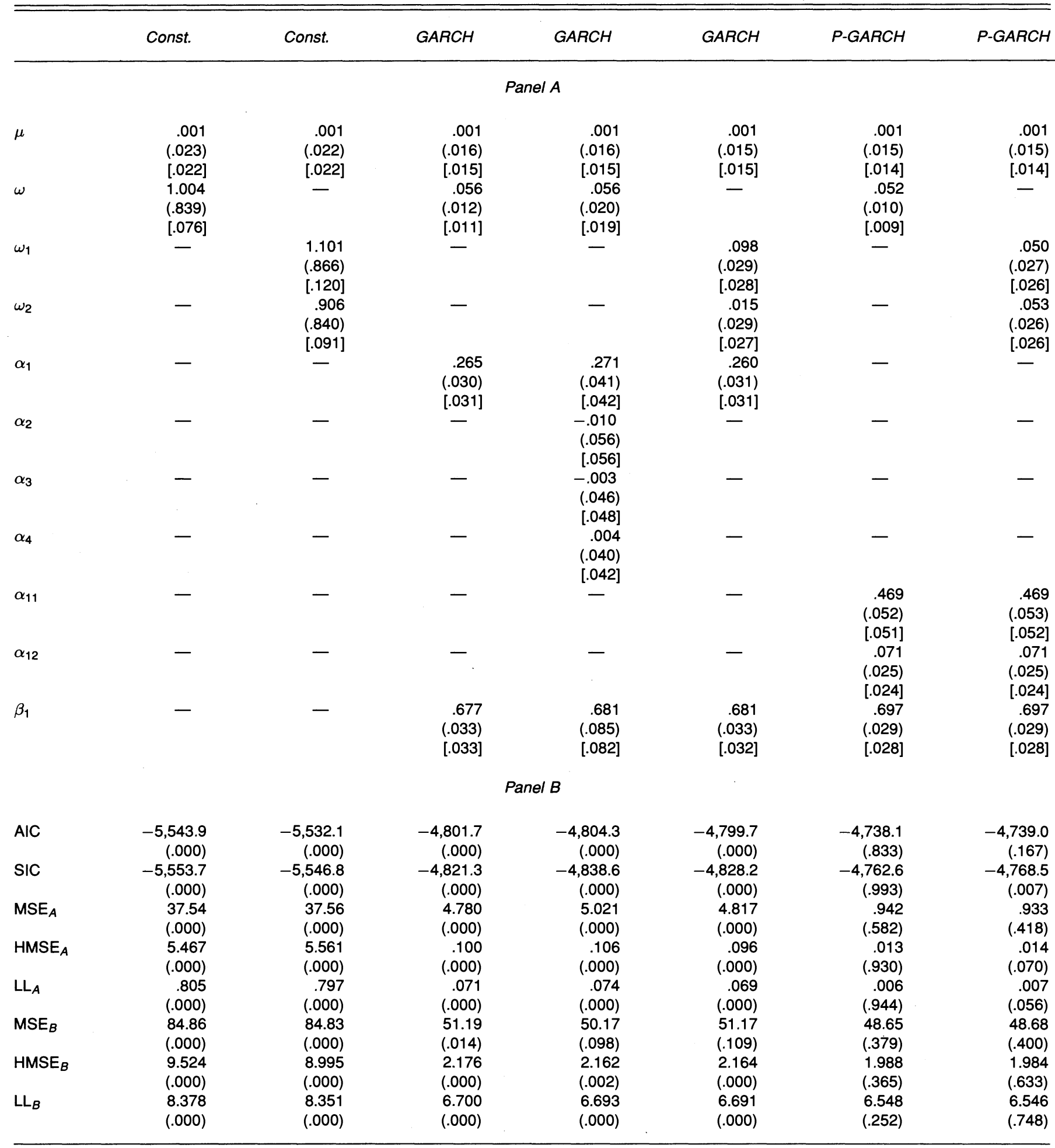

NOTE: The true DGP is $y_{t}=\mu+\tilde{\varepsilon}_{t}, \tilde{\varepsilon}_{t} \mid \Omega_{t-1}^{s} \sim N\left(0, \tilde{\sigma}_{t}^{2}\right), s=1,2, T=1,2, \ldots, 2,000, \tilde{\sigma}_{t}^{2}=\omega+\omega_{s(t)}+\alpha_{1} \tilde{\varepsilon}_{t-1}^{2}+\cdots+\alpha_{4} \tilde{\varepsilon}_{t-4}^{2}+\alpha_{1 s(t)} \tilde{\varepsilon}_{t-1}^{2}+\beta_{1} \tilde{\sigma}_{t-1}^{2}, \mu=.0, \omega=.05, \omega_{1}=\omega_{2}=$ $.0, \alpha_{1}=\cdots=\alpha_{4}=.0, \alpha_{11}=.4666, \alpha_{12}=.0727, \beta_{1}=.7$. Panel A reports the mean of the MLE's and the QMLE across the $N=1,000$ Monte Carlo replications under the P-GARCH(1, 1) DGP. The sample standard deviations for the 1,000 estimates are reported in parentheses, with the mean of the corresponding robust standard error estimates in square brackets. Panel $B$ gives the average value of the Akaike information criterion (AIC) and Schwartz information criterion (SIC) for each of the different model specifications. MSE ${ }_{A}$ and MSE $E_{B}$ denote the average mean squared error for the true conditional variance and squared innovations; that is, $N^{-1} T^{-1} \Sigma_{i=1, N^{2}} \Sigma_{t=1, T}\left[\tilde{\sigma}_{t, i}^{2}\left(\theta_{0}\right)-\tilde{\sigma}_{t, i}^{2}\left(\hat{\theta}_{T, i}\right)\right]^{2}$ and $N^{-1} T^{-1} \Sigma_{i=1, N} \Sigma_{t=1, T}\left[\tilde{\varepsilon}_{t, i}^{2}\left(\theta_{0}\right)-\tilde{\sigma}_{t, i}^{2}\left(\hat{\theta}_{T, i}\right)\right]^{2}$, respectively, where $i$ indicates the order of the replication. $\mathrm{HMSE}_{A}$ and $\mathrm{HMSE}_{B}$ refer to the corresponding heteroscedasticity-adjusted MSE; that is, $N^{-1} T^{-1} \Sigma_{i=1, N^{2}} \Sigma_{t=1, T}\left[\tilde{\sigma}_{t, i}^{2}\left(\theta_{0}\right) \tilde{\sigma}_{t, i}^{2}\left(\hat{\theta}_{T, i}\right){ }^{-1}-1\right]^{2}$ and $N^{-1} T^{-1} \Sigma_{i=1, N} \Sigma_{t=1, T}\left[\tilde{\varepsilon}_{t, i}^{2}\left(\theta_{0}\right) \tilde{\sigma}_{t, i}^{2}\left(\hat{\theta}_{T, i}\right)^{-1}-1\right]^{2}$. The logarithmic loss functions $L_{A}$ and $L_{B}$ are calculated as $N^{-1} T^{-1} \Sigma_{i=1, N^{\prime}} \Sigma_{t=1, T^{\prime}} \ln \left[\tilde{\sigma}_{t, i}^{2}\left(\theta_{0}\right) \tilde{\sigma}_{t, i}^{2}\left(\hat{\theta}_{T, i}\right)-1\right]^{2}$ and $N^{-1} T^{-1} \Sigma_{i=1, N^{\prime}} \Sigma_{t=1, T^{l n}}$ $\left[\tilde{\varepsilon}_{t, i}^{2}\left(\theta_{0}\right) \tilde{\sigma}_{t, i}^{2}\left(\hat{\theta}_{T, i}\right)^{-1}\right]^{2}$, respectively. The proportion of times that a specification was favored by one of the information criteria or a particular loss function are reported in parentheses.

to .096 for the nonperiodic GARCH model with a seasonal dummy in the conditional-variance equation. Ignoring the conditional heteroscedasticity results in a substan- tially larger average loss of $\mathrm{HMSE}_{A}=5.561$. Similar findings are obtained for the logarithmic loss function, $\mathrm{LL}_{A}=N^{-1} T^{-1} \Sigma_{i=1, \ldots, N} \Sigma_{t=1, \ldots, T} \ln \left[\tilde{\sigma}_{t, i}^{2}\left(\theta_{0}\right) \tilde{\sigma}_{t, i}^{2}\left(\hat{\theta}_{T, i}\right)^{-1}\right]^{2}$, 
which assigns proportionally higher weights to wrong predictions in low-variance regimes.

In practice the true conditional variance is unknown, so none of the three loss functions discussed previously is directly applicable. By replacing the true conditional variance with the realized squared residuals, however, the MSE analog becomes $\mathrm{MSE}_{B}=$ $N^{-1} T^{-1} \Sigma_{i=1, \ldots, N} \Sigma_{t=1, \ldots, T}\left[\tilde{\varepsilon}_{t, i}^{2}\left(\theta_{0}\right)-\tilde{\sigma}_{t, i}^{2}\left(\hat{\theta}_{T, i}\right)\right]^{2}$. This is the criterion used by West and Cho (1995) in their evaluation of alternative conditional-variance models for exchange rates, whereas Pagan and Schwert (1990) employed the same $\mathrm{MSE}_{B}$ along with the $\mathrm{LL}_{B}$ loss function defined later in their analysis of competing specifications for stock-market volatility. Interestingly, judged by the $\mathrm{MSE}_{B}$ criterion the three nonperiodic weak GARCH models and the true P-GARCH models all result in fairly similar average squared error losses, although the two PGARCH models yield the smallest loss among the different specifications for $37.9 \%$ and $40.0 \%$ of the replications, respectively. As noted previously, the use of an MSE-type loss function in a heteroscedastic environment is somewhat problematic, however. In particular, by the $\mathrm{MSE}_{B}$ criterion a nonlinear least squares regression of the squared residuals on all the variables in the time $t-1$ information set will always produce the lowest possible in-sample loss. With this in mind, it is interesting to note that the heteroscedasticity-adjusted MSE, $\mathrm{HMSE}_{B}=$ $N^{-1} T^{-1} \Sigma_{i=1, \ldots, N} \Sigma_{t=1, \ldots, T}\left[\tilde{\varepsilon}_{t, i}^{2}\left(\theta_{0}\right) \tilde{\sigma}_{t, i}^{2}\left(\hat{\theta}_{T, i}\right)^{-1}-1\right]^{2}$, almost unambiguously favors the P-GARCH specifications. The average $\mathrm{HMSE}_{B}$ for the estimated P-GARCH models is just slightly below its implied value of 2 , whereas the smallest average value for the three weak GARCH models is 2.162. The findings for the logarithmic loss function, $\mathrm{LL}_{B}=N^{-1} T^{-1} \Sigma_{i=1, \ldots, N} \Sigma_{t=1, \ldots, T} \ln \left[\tilde{\varepsilon}_{t, i}^{2}\left(\theta_{0}\right) \tilde{\sigma}_{t, i}^{2}\left(\hat{\theta}_{T, i}\right)^{-1}\right]^{2}$, are again comparable.

Summing up, the simulation evidence for the P$\operatorname{GARCH}(1,1)$ DGP reported in Table 1 and similar results for other DGP's, available on request, confirm the reliability of the MLE-based inference procedures in the P-GARCH context. Although it is difficult to explicitly quantify in terms of a single measure, the simulations also illustrate that the informational loss associated with the QMLE of a nonperiodic GARCH specification may be quite substantial.

\section{EMPIRICAL EXAMPLES}

To illustrate the empirical relevance of the new $P$ GARCH class of models, this section presents estimation results for two different foreign-exchange-rate series. The first application involves an investigation of the nontradingday effect based on an eight-year-long time series of daily Deutschemark/British pound exchange rates, whereas the second example provides an analysis of the purely repetitive seasonal pattern in a one-year time series consisting of two Deutschemark/U.S. dollar returns per day.

\subsection{Daily Deutschemark/British Pound Exchange-Rate Volatility}

Several recent studies have found that the volatility of daily U.S. dollar exchange rates tends to be highly persistent and well approximated by an integrated or longmemory-type GARCH process; for example, see Engle and Bollerslev (1986), McCurdy and Morgan (1988), Baillie and Bollerslev (1989), Hsieh (1989), Baillie et al. (in press), and Taylor (1994). At the same time, Bollerslev and Engle (1993) argued that, even though the volatility processes for the daily Deutschemark/U.S. dollar and the British pound/U.S. dollar exchange rates are both highly persistent, it appears that the nonstationarity is common across the two rates so that the volatility of the corresponding bilateral Deutschemark/British pound rate shows less persistence. Thus, to circumvent issues related to the appropriate modeling of the long-run volatility persistence, we shall here concentrate on the dynamics of the Deutschemark/British pound exchange rate. The data are constructed from the corresponding daily U.S. dollar rates recorded by the International Monetary Fund in International Financial Statistics and cover the period from January 3, 1984, through December 31, 1991, for a total of 1,974 observations.

Following standard practice, define the daily percentage nominal returns,

$$
y_{t} \equiv 100 \cdot\left[\ln \left(P_{t}\right)-\ln \left(P_{t-1}\right)\right]=\mu+\tilde{\varepsilon}_{t},
$$

where $P_{t}$ denotes the bilateral spot exchange rate for $t=$ $0,1, \ldots, 1,974$. The first-order sample autocorrelation coefficient for the returns equals only .009. The insignificant Ljung and Box (1978) portmanteau test for up to twentiethorder serial correlation in $\tilde{\varepsilon}_{t}\left(\hat{\theta}_{T}\right)$ reported in the first column of Table 2, $Q(20)$, also does not reject that $\left\{y_{t}\right\}$ is an approximate martingale process. Note that the standard portmanteau tests for serial correlation in the mean tend to be very conservative in the presence of ARCH effects; see Diebold (1988) and Bollerslev and Mikkelsen (in press). To investigate the influence of market closure, the next two columns of the table report the estimates from two simple periodic mean and variance formulations, $\mu+\mu_{s(t)}$ and $\omega+\omega_{s(t)}$, in which the stage of the periodic cycle equals 1 on Mondays and other days following no trading in the Deutschemark or British pound/U.S. dollar market during regular European trading hours and 0 otherwise. The sample consists of 456 such nontrading periods, which corresponds to roughly $23 \%$ of the observations. For ease of interpretation we restrict $\mu_{0} \equiv 0$ and $\omega_{0} \equiv 0$. Whereas no systematic mean effect is forthcoming, the estimate for the periodic nontrading-day effect in the variance, $\omega_{1}$, is highly significant, and suggests an average increase in the volatility of about $50 \%$. At the same time, the large value of the $Q^{2}(20)$ portmanteau test for the squared standardized residuals, $\tilde{\varepsilon}_{t}^{2}\left(\hat{\theta}_{T}\right) \tilde{\sigma}_{t}^{2}\left(\hat{\theta}_{T}\right)^{-1}$, indicates that very pronounced volatility clustering remains. The last three rows of the table therefore report the QMLE for a simple $\operatorname{GARCH}(1,1)$ model, a $\operatorname{GARCH}(1,1)$ model that includes the seasonal nontrading-day dummy in the conditional-variance equation, and a P-GARCH $(1,1)$ model. Following the analysis 
Table 2. P-GARCH Models for Daily Deutschemark/British Pound Exchange Rate

\begin{tabular}{|c|c|c|c|c|c|c|}
\hline & Const. & Const. & Const. & $G A R C H$ & $G A R C H$ & $P-G A R C H$ \\
\hline$\mu$ & $\begin{array}{r}-.016 \\
(.011)\end{array}$ & $\begin{array}{r}-.013 \\
(.011)\end{array}$ & $\begin{array}{r}-.015 \\
(.010)\end{array}$ & $\begin{array}{r}-.006 \\
(.009)\end{array}$ & $\begin{array}{l}-.009 \\
(.008)\end{array}$ & $\begin{array}{r}-.006 \\
(.008)\end{array}$ \\
\hline$\mu_{1}$ & - & $\begin{array}{l}-.017 \\
(.028)\end{array}$ & - & - & - & - \\
\hline$\omega$ & $\begin{array}{r}.221 \\
(.012)\end{array}$ & $\begin{array}{r}.200 \\
(.011)\end{array}$ & $\begin{array}{r}.200 \\
(.011)\end{array}$ & $\begin{array}{r}.264 \\
(.075)\end{array}$ & $\begin{array}{r}.259 \\
(.086)\end{array}$ & $\begin{array}{r}.341 \\
(.169)\end{array}$ \\
\hline$\omega_{1}$ & - & $\begin{array}{r}.092 \\
(.036)\end{array}$ & $\begin{array}{r}.092 \\
(.036)\end{array}$ & - & $\begin{array}{r}.054 \\
(.022)\end{array}$ & $\begin{array}{r}.043 \\
(.026)\end{array}$ \\
\hline$\alpha_{1}$ & - & - & - & $\begin{array}{r}.153 \\
(.054)\end{array}$ & $\begin{array}{r}.137 \\
(.035)\end{array}$ & $\begin{array}{r}.178 \\
(.043)\end{array}$ \\
\hline$\alpha_{11}$ & - & - & - & - & - & $\begin{array}{r}-.111 \\
(.044)\end{array}$ \\
\hline$\beta_{1}$ & - & - & - & $\begin{array}{r}.806 \\
(.073)\end{array}$ & $\begin{array}{r}.832 \\
(.044)\end{array}$ & $\begin{array}{r}.822 \\
(.042)\end{array}$ \\
\hline AIC & $-2,626.2$ & $-2,602.7$ & $-2,601.1$ & $-2,221.2$ & $-2,191.0$ & $-2,179.3$ \\
\hline SIC & $-2,652.5$ & $-2,625.1$ & $-2,617.9$ & $-2,243.5$ & $-2,219.0$ & $-2,21.2 .9$ \\
\hline$b_{3}$ & -.25 & -.25 & -.25 & -.40 & -.44 & -.46 \\
\hline$b_{4}$ & 6.63 & 6.18 & 6.18 & 6.56 & 5.89 & 5.87 \\
\hline$Q(20)$ & 27.8 & 27.2 & 26.9 & 19.3 & 18.8 & 19.1 \\
\hline$Q^{2}(20)$ & 507.6 & 593.0 & 599.4 & 17.5 & 21.7 & 23.9 \\
\hline MSE & .275 & .273 & .273 & .253 & .252 & .257 \\
\hline HMSE & 5.63 & 5.18 & 5.18 & 5.52 & 4.88 & 4.67 \\
\hline LL & 9.67 & 9.87 & 9.52 & 8.79 & 8.21 & 8.66 \\
\hline
\end{tabular}

NOTE: The table reports the QMLE's for the daily percentage returns on the Deutschemark/British pound exchange rate from January 3 , 1984, through December 31, 1991, for a total of 1,974 observations. Robust standard errors are reported in parentheses. The estimated models are $y_{t} \equiv 100 \cdot\left[\ln \left(P_{t}\right)-\ln \left(P_{t-1}\right)\right]=\mu+\mu_{s(t)}+\tilde{\varepsilon}_{t}, \tilde{\varepsilon}_{t} \mid \Omega_{t-1}^{s} \sim N\left(0, \tilde{\sigma}_{t}^{2}\right), s=0,1, T=1,2, \ldots, 1974, \tilde{\sigma}_{t}^{2}=\omega+\omega_{s(t)}-$ $\left(\omega+\omega_{s(t-1)}\right)\left(\alpha_{1}+\beta_{1}+\alpha_{1 s(t)}\right)+\left(\alpha_{1}+\alpha_{1 s(t)}\right) \tilde{\varepsilon}_{t-1}^{2}+\beta_{1} \tilde{\sigma}_{t-1}^{2}$. The value of the corresponding Akaike and Schwartz information criteria are given in the AIC and SIC rows. The sample skewness and kurtosis for the standardized residuals, $\tilde{\varepsilon}_{t}\left(\hat{\theta}_{T}\right) \tilde{\sigma}_{t}^{2}\left(\hat{\theta}_{T}\right)^{-1 / 2}$, are denoted by $b_{3}$ and $b_{4}$, respectively. $Q(20)$ and $Q^{2}(20)$ refer to the Ljung-Box portmanteau tests for up to twentieth-order serial correlation in the standardized and the squared standardized residuals, respectively. The different loss criteria are calculated as MSE $=T^{-1} \Sigma_{t=1, T}$ $\left[\tilde{\varepsilon}_{t}^{2}\left(\hat{\theta}_{T}\right)-\tilde{\sigma}_{t}^{2}\left(\hat{\theta}_{T}\right)\right]^{2}, \mathrm{HMSE}=T^{-1} \Sigma_{t=1, T}\left[\tilde{\varepsilon}_{t}^{2}\left(\hat{\theta}_{T}\right) \tilde{\sigma}_{t}^{2}\left(\hat{\theta}_{T}\right)^{-1}-1\right]^{2}$, and $\mathrm{LL}=T^{-1} \Sigma_{t=1, T} \ln \left[\tilde{\varepsilon}_{t}^{2}\left(\hat{\theta}_{T}\right) \tilde{\sigma}_{t}^{2}\left(\theta_{T}\right)^{-1}\right]^{2}$.

of Baillie and Bollerslev (1989), the seasonal dummy variable is entered in the conditional-variance equation to allow for an impulse effect,

$$
\begin{aligned}
\tilde{\sigma}_{t}^{2}=\omega+\omega_{s(t)}-(\omega+ & \left.\omega_{s(t-1)}\right)\left(\alpha_{1}+\beta_{1}+\alpha_{1 s(t)}\right) \\
& +\left(\alpha_{1}+\alpha_{1 s(t)}\right) \tilde{\varepsilon}_{t-1}^{2}+\beta_{1} \tilde{\sigma}_{t-1}^{2},
\end{aligned}
$$

where by definition $\omega_{0} \equiv 0$ and $\alpha_{10} \equiv 0$. Although the $\operatorname{GARCH}(1,1)$ model parsimoniously captures the own temporal dependence in the second-order moments of the returns, the nontrading-day effect remains very significant. Both the AIC and the SIC strongly favor the inclusion of the seasonal dummy variable, $\omega_{1}$, in the conditional-variance equation. The same information criteria, however, as well as the robust $t$ statistic for the $\alpha_{11}$ parameter estimate from the P-GARCH $(1,1)$ model, suggest the importance of allowing for a richer dynamic periodic structure in characterizing the nontrading-day effects. Interestingly, the sum of the estimated autoregressive coefficients for the P-GARCH model when $s(t)=0$ equals $\hat{\alpha}_{1}+\hat{\beta}_{1}=1.000$, whereas on days following market closures $\hat{\alpha}_{1}+\hat{\alpha}_{11}+\hat{\beta}_{1}=.889$. Thus, shocks to the conditional variance that occur when the market is closed appear to be less informative about the future volatility and tend to die out at a faster rate than shocks that occur during normal trading days. As discussed in Section 2, the gain obtained by allowing for the richer P-GARCH structure will ultimately depend on the particu- lar application of the model. It is noteworthy, however, that the heteroscedasticity-adjusted MSE criterion advocated in Section 2 is also minimized for the P-GARCH specification.

\subsection{Intraday Deutschemark/U.S. Dollar Exchange-Rate Volatility}

The increased availability of high-frequency financial data has stimulated a growing research interest in the complex intraday-return dynamics. Recent examples from the foreign-exchange market include those of Baillie and Bollerslev (1991), Bollerslev and Domowitz (1993), Engle, Ito, and Lin (1990), Müller et al. (1990), and Dacorogna et al. (1993) among others. The purpose of the present empirical example is not to add to this existing literature per se but merely to illustrate the potential benefits offered by the new P-GARCH class of models in this context.

The foreign-exchange market operates on a continuous 24-hours-a-day basis. We shall focus on a time series of returns observed only twice a day, however. Specifically, the first return for the day gives the logarithmic price change over the seven most active trading hours in the Deutschemark/U.S. dollar exchange-rate market from 8:00 Greenwich Mean Time (GMT) through 15:00 GMT. The next return observation covers the 17-hour interval from 15:00 GMT through 8:00 GMT the following day. This particular definition of the return intervals is motivated by the intraday volatility patterns documented by Baillie and Bollerslev (1991) and Müller et al. (1990) and ensures that the two corresponding unconditional sample variances are 
Table 3. P-GARCH Models for Intraday Deutschemark/U.S. Dollar Exchange Rate

\begin{tabular}{|c|c|c|c|c|c|c|c|}
\hline & Const. & AR const. & $P-A R$ & $P-A R$ & $\begin{array}{c}P-A R \\
G A R C H\end{array}$ & $\begin{array}{c}P-A R \\
P-G A R C H\end{array}$ & $\begin{array}{c}P-A R \\
P-G A R C H\end{array}$ \\
\hline$\mu$ & $\begin{array}{r}.023 \\
(.022)\end{array}$ & $\begin{array}{r}.014 \\
(.022)\end{array}$ & - & $\begin{array}{r}.023 \\
(.024)\end{array}$ & $\begin{array}{r}.014 \\
(.022)\end{array}$ & $\begin{array}{r}.015 \\
(.024)\end{array}$ & $\begin{array}{r}.016 \\
(.023)\end{array}$ \\
\hline$\mu_{1}$ & - & - & $\begin{array}{r}.020 \\
(.031)\end{array}$ & - & - & - & - \\
\hline$\mu_{2}$ & - & - & $\begin{array}{r}.026 \\
(.032)\end{array}$ & - & - & - & - \\
\hline$\phi_{1}$ & - & $\begin{array}{r}.107 \\
(.042)\end{array}$ & - & - & - & - & - \\
\hline$\phi_{11}$ & - & - & $\begin{array}{r}-.010 \\
(.064)\end{array}$ & - & - & - & - \\
\hline$\phi_{12}$ & - & - & $\begin{array}{r}.194 \\
(.055)\end{array}$ & $\begin{array}{r}.193 \\
(.055)\end{array}$ & $\begin{array}{r}.196 \\
(.050)\end{array}$ & $\begin{array}{r}.218 \\
(.052)\end{array}$ & $\begin{array}{r}.209 \\
(.049)\end{array}$ \\
\hline$\omega$ & $\begin{array}{r}.255 \\
(.020)\end{array}$ & $\begin{array}{r}.238 \\
(.038)\end{array}$ & - & $\begin{array}{r}.251 \\
(.020)\end{array}$ & $\begin{array}{r}.268 \\
(.091)\end{array}$ & $\begin{array}{r}.248 \\
(.024)\end{array}$ & $\begin{array}{r}.249 \\
(.030)\end{array}$ \\
\hline$\omega_{1}$ & - & - & $\begin{array}{r}.247 \\
(.027)\end{array}$ & - & - & - & - \\
\hline$\omega_{2}$ & - & - & $\begin{array}{r}.255 \\
(.029)\end{array}$ & - & - & - & - \\
\hline$\alpha_{1}$ & - & $\begin{array}{r}.030 \\
(.012)\end{array}$ & - & - & $\begin{array}{r}.031 \\
(.015)\end{array}$ & - & - \\
\hline$\alpha_{11}$ & - & - & - & - & - & $\begin{array}{r}.128 \\
(.044)\end{array}$ & $\begin{array}{r}.098 \\
(.046)\end{array}$ \\
\hline$\alpha_{12}$ & - & - & - & - & - & $\begin{array}{r}-.045 \\
(.024)\end{array}$ & - \\
\hline$\beta_{1}$ & - & $\begin{array}{r}.941 \\
(.018)\end{array}$ & - & - & $\begin{array}{r}.944 \\
(.024)\end{array}$ & $\begin{array}{r}.820 \\
(.048)\end{array}$ & $\begin{array}{r}.866 \\
(.048)\end{array}$ \\
\hline AIC & -767.1 & -751.3 & -765.5 & -759.6 & -746.0 & -738.0 & -738.9 \\
\hline SIC & -775.6 & -772.5 & -791.0 & -772.4 & -767.2 & -763.5 & -760.1 \\
\hline$b_{3}$ & .32 & .19 & .34 & .34 & .16 & .16 & .13 \\
\hline$b_{4}$ & 4.09 & 4.02 & 4.26 & 4.27 & 3.99 & 3.89 & 3.94 \\
\hline$Q(20)$ & 27.5 & 24.0 & 22.6 & 22.6 & 23.4 & 24.5 & 24.6 \\
\hline$Q^{2}(20)$ & 61.5 & 23.6 & 61.4 & 63.7 & 24.7 & 26.2 & 25.2 \\
\hline MSE & .202 & .204 & .205 & .206 & .201 & .200 & .199 \\
\hline HMSE & 3.09 & 3.23 & 3.26 & 3.27 & 2.82 & 2.91 & 2.92 \\
\hline LL & 8.25 & 8.27 & 9.53 & 8.93 & 8.83 & 7.95 & 8.13 \\
\hline
\end{tabular}

NOTE: The table reports the QMLE for intraday percentage returns on the Deutschemark/U.S. dollar exchange. The return series consists of two observations per day over the October 1, 1992, through September 29, 1993, period for a total sample size of 518 . The estimated models are $y_{t} \equiv 100 \cdot\left[\ln \left(P_{t}\right)-\ln \left(P_{t-1}\right)\right]=\mu+\mu_{s(t)}-\left(\mu+\mu_{s(t-1)}\right)\left(\phi_{1}+\phi_{1 s(t)}\right)+\left(\phi_{1}+\phi_{1 s(t)}\right) y_{t-1}+\tilde{\varepsilon}_{t}, \tilde{\varepsilon}_{t} \mid \Omega_{t-1}^{s} \sim N\left(0, \tilde{\sigma}_{t}^{2}\right)$, $s=1,2, T=1,2, \ldots, 518, \tilde{\sigma}_{t}^{2}=\omega+\omega_{s(t)}-\left(\omega+\omega_{s(t-1)}\right)\left(\alpha_{1}+\beta_{1}+\alpha_{1 s(t)}\right)+\left(\alpha_{1}+\alpha_{1 s(t)}\right) \tilde{\varepsilon}_{t-1}^{2}+\beta_{1} \tilde{\sigma}_{t-1}^{2}$. For a definition of the statistics reported in the table, see the note to Table 2 .

approximately the same. The continuously recorded bidask quotations used in the calculation of these returns were obtained from Olsen and Associates. The sample period extends from October 1, 1992, through September 29, 1993, for a total of 518 intraday-return observations excluding weekends. For a more detailed description of the data set and method of data capture and outlier filtration, we refer to Andersen and Bollerslev (in press) and Dacorogna et al. (1993).

Although the example in Section 3.1 illustrates the potential importance of allowing for weekend and nontradingday effects, given the much shorter sample period available for the intraday data, we shall here concentrate on modeling the purely repetitive 24 -hour trading-day cycle. The first row in Table 3 gives the unconditional sample mean and variance for the 518 intraday-return observations, along with the same set of summary statistics and information criteria reported in Table 2 . In contrast to the results for the daily returns, the intraday returns appear to be serially correlated with a first-order sample autocorrelation coefficient of .088. Although the simple nonperiodic AR(1)-
$\operatorname{GARCH}(1,1)$ model reported in the second column of the table captures most of the serial correlation in the first- and second-order moments of the returns, the model makes no distinction between the returns for the most active European trading hours, $s(t)=1$, and the returns for the remainder of the day, $s(t)=2$. From the estimated P-AR(1) model in the third column of the table, however,

$$
\begin{aligned}
y_{t} & \equiv 100 \cdot\left[\ln \left(P_{t}\right)-\ln \left(P_{t-1}\right)\right] \\
& =\mu+\mu_{s(t)}-\left(\mu+\mu_{s(t-1)}\right) \phi_{1 s(t)}+\phi_{1 s(t)} y_{t-1}+\tilde{\varepsilon}_{t},
\end{aligned}
$$

only $\phi_{12}$ is significantly different from 0 . News that occurs during European trading hours gets incorporated into the price with a lag during the rest of the day, whereas there is no evidence for any lagged dependence in the returns for the active European segment of the market. The estimates for $\omega_{1}$ and $\omega_{2}$ and the results for the restricted P-AR(1) model in the fourth column with $\omega_{1}=\omega_{2}=\omega$ also confirm that, in spite of the different calendar length of the two return intervals, the particular segmentation of the 24-hour trading day employed here ensures that the two 
corresponding intraday sample variances are approximately the same. Not surprisingly, there is no evidence for any systematic U.S. dollar depreciation or appreciation during specific times of the day as $\hat{\mu}_{1} \approx 0$ and $\hat{\mu}_{2} \approx 0$. Whereas the $Q^{2}(20)$ statistic for the residuals from the P-AR(1) model remains highly significant, the portmanteau statistics for the P-AR(1)-GARCH $(1,1)$ model in the next column indicate that this model captures most of the own serial dependence in the returns. By allowing for a periodic structure in the conditional-variance equation, however, the P-GARCH(1, 1) models reported in the last two columns of the table indicate that only shocks that originate during the less active time of the market significantly increase the overall volatility; that is, only $\alpha_{11}>0$. Note, that the richer P-GARCH $(1$, 1) formulations are strongly preferred by both of the two model-selection criteria. It is certainly possible that by also incorporating the effect of market closure and other timevarying components an even better representation of the intraday-return dynamics could be obtained. The model estimates reported in Table 3, however, suggest that any future research along these lines may successfully exploit periodic ARCH-type structures.

\section{CONCLUDING REMARKS}

The discrete-time periodic GARCH class of models proposed in this article provides a natural generalization of time-invariant seasonal GARCH models to allow for a greater degree of flexibility when modeling periodicity in the conditional variances of speculative prices. The reported simulation evidence and empirical examples illustrate the feasibility and practical relevance of allowing for varying dynamic responses across the different stages of the periodic cycle. Of course, the idea of using periodic structures in formulating time series models for conditional heteroscedasticity is not limited to the GARCH class of models. Of the many possible univariate and multivariate extensions, the asymmetric P-EGARCH model discussed briefly in Section 1 may prove particularly useful in the future estimation of more accurate models for high-frequency stockmarket volatility.

In conclusion, we would like to draw attention to a time-deformation interpretation of the P-ARCH class of models. The original idea of time deformation, or subordinate stochastic processes, advanced by Clark (1973) and Tauchen and Pitts (1983), ties the asset price movements to the number of market transactions, trading volume, or other measures of market activity; for a recent analysis along these lines, see Andersen (in press). Taking the time deformation to be purely deterministic and only dependent on the periodic cycle, however, naturally leads to a P-ARCH-type model. In particular, suppose that the returns at some high-frequency operational, or market, time scale are generated by a $\operatorname{GARCH}(1,1)$ model with parameters $\omega, \alpha_{1}$, and $\beta_{1}$. Then, from the aggregation results of Drost and Nijman (1993), discussed in Section 1, it follows that, when aggregated over $m$ periods, the parameters of the corresponding weak $\operatorname{GARCH}(1,1)$ model are $\omega_{m}=m \omega\left[1-\left(\alpha_{1}+\beta_{1}\right)^{m}\right]\left[1-\left(\alpha_{1}+\beta_{1}\right)\right]^{-1}$ and $\alpha_{m}=\left(\alpha_{1}+\beta_{1}\right)^{m}-\beta_{m}$, where $\beta_{m}$ is a complicated function of $m, \alpha_{1}, \beta_{1}$, and the conditional kurtosis of the highfrequency process. Now suppose that the observed process in calendar time is characterized by a deterministic periodic cycle of length $S$ so that each stage of the cycle is associated with a different level of aggregation from the operational time scale to the regularly spaced calendar-time observation interval; that is, to each $s=1,2, \ldots, S$ corresponds a different aggregation level $m(s)$. The P-GARCH $(1,1)$ specification then becomes immediately apparent by setting $\omega_{s(t)}=\omega_{m(s)}, \alpha_{1 s(t)}=\alpha_{m(s)}$, and $\beta_{1 s(t)}=\beta_{m(s)}$. Thus, a periodic cycle corresponding to different levels of aggregation that reflects the cyclical behavior of market activity yields a simple interpretation of the P-GARCH model as a process subject to periodic time deformation.

This same idea can be rendered more sophisticated. In particular, although the discrete-time stochastic ARCH-type difference equations have found very wide empirical use, continuous-time stochastic differential equations have often proven more convenient in the analysis of theoretical pricing models. Similarly, notions of time deformation have traditionally been analyzed in a continuous-time setting. We shall not pursue this analysis at any great length here. We note, however, that the discrete time-aggregation arguments presented previously could be extended to continuous time by using the diffusion approximation for the weak $\operatorname{GARCH}(1,1)$ model developed by Drost and Werker (in press). Alternatively, Ghysels and Jasiak (1994) recently proposed a direct extension of the standard stochastic volatility model subject to time deformation. According to this specification, the returns process evolves in the regularly spaced calendar time, $t$, but the evolution of the instantaneous volatility is determined by an Ornstein-Uhlenbeck process in operational time, $\tau$; that is, $d\left[\ln \left(P_{t}\right)\right]=\mu_{t} d t$ $+\sigma_{t} d W_{1 t}$ and $d\left[\ln \left(\sigma_{\tau}^{2}\right)\right]=-\beta\left[\ln \left(\sigma_{\tau}^{2}\right)-\alpha\right] d \tau+d W_{2 \tau}$, where $W_{1 t}$ and $W_{2 t}$. denote independent standard Wiener processes. Note that in the absence of any time deformation so that $\tau \equiv t$ this model corresponds to the diffusion limit of the EGARCH model derived by Nelson (1990). Assuming, however, that the operational time scale is measurable with respect to the usual calendar-time filtration, $\tau \equiv g(t)$, and that the time deformation is purely deterministic and only dependent on the periodic cycle, it is possible to show that the corresponding discrete-time process for the conditional variance reduces to a P-ARCH-type model; see Ghysels, Gouriéroux, and Jasiak (1995) for a review of the relevant stochastic process theory. We leave further theoretical developments and empirical work along these lines for future research.

\section{ACKNOWLEDGMENTS}

We thank Richard T. Baillie for helpful discussions. The article has also benefited greatly from the very constructive and helpful comments by the editor, George Tauchen, the associate editor, and two anonymous referees. The second author acknowledges the financial support from Natural Sciences and Engineering Research Council (NSERC) and 
Social Sciences and Humanities Research Council (SSHRC) of Canada and the Fonds pour la Formation de Chercheurs et l'Aide a la Recherche (FCAR) of Quebec.

\section{APPENDIX: TIME INVARIANT WEAK GARCH REPRESENTATION OF THE P-GARCH MODEL}

In this appendix we provide an illustration of the techniques that may be used to implicitly characterize the mapping between a P-GARCH model with a fixed periodic cycle of length $S$ and its time-invariant weak GARCH representation. Formally this operation is most easily accomplished by constructing a skip-sampled vector representation of the squared residuals that collects all the observations over a single periodic cycle; that is, $\tilde{\varepsilon}_{\tau} \equiv\left(\tilde{\varepsilon}_{S \tau}^{2}, \tilde{\varepsilon}_{S(\tau-1)+S-1}^{2}, \ldots, \tilde{\varepsilon}_{S(\tau-1)+2}^{2}, \tilde{\varepsilon}_{S(\tau-1)+1}^{2}\right)$, where $\tau$ refers to the time index for the $\left\{\tilde{\varepsilon}_{\tau}\right\}$ process. For instance, in applications of periodic ARMA models to quarterly or monthly data, $\tau$ could be an annual time index. In the application of periodic ARCH processes to daily or intraday sampling frequencies, $\tau$ may correspond to a weekly time scale with a vector representation of daily series or to the daily sampling of a vector of hourly processes. Similarly, define $\tilde{\boldsymbol{\eta}}_{\tau}$ as the $S \times 1$ vector of innovations that appear in the weak P-GARCH$(p, q)$ model in Equation (8). Because the vectors obtained in this way cover an entire periodic cycle, they encompass all possible parameter variations. Specifically, consider the simple case in which $S=2$ with alternating periods, each of which obey a $\operatorname{GARCH}(1,1)$ structure. Then from Equation (8), the following bivariate vector system becomes apparent:

$$
\begin{aligned}
\left(\begin{array}{cc}
1 & -\left(\alpha_{12}+\beta_{12}\right) \\
0 & 1
\end{array}\right)\left(\begin{array}{c}
\tilde{\varepsilon}_{2 \tau}^{2} \\
\tilde{\varepsilon}_{2 \tau-1}^{2}
\end{array}\right) \\
=\left(\begin{array}{c}
\omega_{2} \\
\omega_{1}
\end{array}\right)+\left(\begin{array}{cc}
0 & 0 \\
\alpha_{11}+\beta_{11} & 0
\end{array}\right)\left(\begin{array}{c}
\tilde{\varepsilon}_{2(\tau-1)}^{2} \\
\tilde{\varepsilon}_{2(\tau-1)-1}^{2}
\end{array}\right) \\
\quad+\left(\begin{array}{cc}
1 & -\beta_{12} \\
0 & 1
\end{array}\right)\left(\begin{array}{c}
\tilde{\eta}_{2 \tau} \\
\tilde{\eta}_{2 \tau-1}
\end{array}\right) \\
\quad+\left(\begin{array}{cc}
0 & 0 \\
-\beta_{11} & 0
\end{array}\right)\left(\begin{array}{c}
\tilde{\eta}_{2(\tau-1)} \\
\tilde{\eta}_{2(\tau-1)-1}
\end{array}\right)
\end{aligned}
$$

or, more compactly,

$$
\begin{aligned}
\tilde{\varepsilon}_{\tau}^{2}= & \left(\begin{array}{c}
\omega_{2}+\left(\alpha_{12}+\beta_{12}\right) \omega_{1} \\
\omega_{1}
\end{array}\right) \\
& +\left(\begin{array}{cc}
\left(\alpha_{11}+\beta_{11}\right)\left(\alpha_{12}+\beta_{12}\right) & 0 \\
\alpha_{11}+\beta_{11} & 0
\end{array}\right) \tilde{\varepsilon}_{\tau-1}^{2} \\
& +\left(\begin{array}{cc}
1 & \alpha_{12} \\
0 & 1
\end{array}\right) \tilde{\eta}_{\tau}+\left(\begin{array}{cc}
-\beta_{11}\left(\alpha_{12}+\beta_{12}\right) & 0 \\
-\beta_{11} & 0
\end{array}\right) \tilde{\eta}_{t \tau-1} .
\end{aligned}
$$

This equation defines a time-invariant representation for the $\left\{\tilde{\varepsilon}_{\tau}^{2}\right\}$ process. Thus, by straightforward arguments, if $\left(\alpha_{11}+\beta_{11}\right)\left(\alpha_{12}+\beta_{12}\right)<1$ so that the eigenvalues for the first-order autoregressive lag matrix are less than 1, the unconditional variance of the process is finite. Note that, unlike the standard multivariate ARCH representations surveyed by Bollerslev et al. (1994), the vector ARCH process in (A.2) does not involve any conditional cross-covariances because each element of the $\left\{\tilde{\varepsilon}_{\tau}^{2}\right\}$ vector process represents the same underlying univariate process sampled at a different points in time.

Of course, these arguments are not constrained to the P$\operatorname{GARCH}(1,1)$ model with $S=2$. Under suitable regularity conditions ensuring that the roots of the characteristic autoregressive polynomial for the corresponding vector system have modules greater than 1 , any weak $\operatorname{P-GARCH}(p, q)$ process may be expressed in terms of its fundamental timeinvariant Wold decomposition,

$$
\tilde{\boldsymbol{\varepsilon}}_{\tau}^{2}=\boldsymbol{\omega}+\sum_{j=0}^{\infty} \mathbf{A}_{j} \tilde{\boldsymbol{\eta}}_{\tau-j} \equiv \boldsymbol{\omega}+\mathbf{A}(L) \tilde{\boldsymbol{\eta}}_{\tau},
$$

where the elements of the $\boldsymbol{\omega}$ vector and $\mathbf{A}_{j}$ coefficient matrices are determined by the polynomials for the underlying model. From Equation (A.3), the multivariate covariance generating function and spectral representation may then be written as

$$
F\left(e^{-i \cdot z}\right)=\mathbf{A}\left(e^{-i \cdot z}\right) Q \mathbf{A}\left(e^{i \cdot z}\right)^{\prime}
$$

where $-\pi \leq z \leq \pi$ and $Q$ denotes the unconditional covariance matrix for the $S$-dimensional innovation process, $\left\{\tilde{\boldsymbol{\eta}}_{\tau}\right\}$. Now, following Tiao and Grupe (1980), it is possible to show that there exist parameters $\sigma_{\eta}^{2}, \alpha_{1}, \ldots, \alpha_{P}$ and $\beta_{1}, \ldots, \beta_{Q}$ such that

$$
\begin{aligned}
\sigma_{\eta}^{2}\left[\frac{\sum_{j=1}^{\max (P, Q)}\left(\alpha_{j}+\beta_{j}\right) e^{-i \cdot z \cdot j}}{1-\sum_{j=1}^{Q} \beta_{j} e^{-i \cdot z \cdot j}}\right]^{2} \\
=R\left(e^{-i \cdot z}\right) F\left(e^{-S \cdot i \cdot z}\right) R\left(e^{-i \cdot z}\right)^{\prime},
\end{aligned}
$$

where $R\left(e^{-i \cdot z}\right)=S^{1 / 2}\left(1, e^{-i \cdot z}, \ldots, e^{-(S-1) \cdot i \cdot z}\right)$. Equation (A.5) therefore establishes an indirect relationship between the parameters for the $\operatorname{P-GARCH}(p, q)$ model and the corresponding weak $\operatorname{GARCH}(P, Q)$ process with parameters $\alpha_{i}$ and $\beta_{j}$ for $i=1, \ldots, P$ and $j=1, \ldots, Q$. Unfortunately, this relationship is only implicit and does not lend itself to any analytical characterization of the actual weak $\operatorname{GARCH}(P, Q)$ parameters. It is possible to show, however, that the autoregressive order of the time-invariant weak GARCH process necessarily exceeds the order of the periodic cycle; that is, $P \geq S$ whenever $p \neq 0$. For further discussion along these lines within the context of periodic ARMA processes with a fixed repetitive cycle, see Osborn (1991).

As previously noted, in many financial applications the periodic cycle is not purely repetitive, but it is rather repetitive with some upperbound, $S$. For example, the upperbound $S=5$ associated with the nontrading-day cycle is not attained every week. In such cases, the simplicity afforded by the skip-sampled vector representation illustrated in (A.1) is no longer available because the varying phase 
of the cycle precludes the use of stacked data vectors of fixed length. Hence, the elegance of the multivariate representation appearing in (A.3) and its spectral counterpart cannot be exploited. The basic principles underlying the Tiao-Grupe formula operations remain applicable, however, although without the simplicity summarized by a simple formula. Most importantly, the mapping from a periodic ARMA, or weak GARCH, model to its corresponding seasonal fixed parameter representation is still based on foregoing the seasonal conditioning. In particular, the first and second unconditional moments of the process can alway:be computed from averaging the first and second moments conditional on the period if the averaging is done over all stages of the cycle. Likewise, the same idea applies to the entire autocorrelation structure. These operations are valid both with purely repetitive and predetermined nonrepetitive periodic cycles. Of course in the latter case the computations are more involved because there is no longer a simple repetitive accounting rule for assigning each data point to a particular stage of the periodic cycle.

The asymptotic distribution results in Section 2 obviously allow for the calculation of standard Wald, Lagrange multiplier, and likelihood ratio tests in an MLE, or QMLE, context. In some applications, however, it may be of interest to test for periodicity without an explicit model speci- $\rightarrow$ fication. When the source of the periodic variation is nonrepetitive and not directly observable this can be quite involved. For periodic ARMA structures, Hansen and Sargen (1990, chapter 10, app. A.2) provided an elaborate discussion of the theoretical underpinnings that would justify the construction of such tests. A more detailed discussion of their framework would require a fairly technical elaboration of the stochastic-process theory of periodic structures because their representation is based on notions of ergodicity and invariant sets associated with measure-preserving mappings defined on Borel $\sigma$ algebras. Suffice it to say that the Hansen-Sargent framework could in principle be considered for the weak GARCH processes introduced in this article also. This, however, would require regularity conditions, such as the existence of higher-order moments, that may be harder to maintain in the ARCH context.

\section{[Received March 1994. Revised September 1994.]}

\section{REFERENCES}

Akaike, H. (1973), "Information Theory and an Extension of the Maximun Likelihood Principle," in Second International Symposium on Information Theory, eds. B. N. Petrov and F. Csáki, Budapest: Akadémiai Kiadó pp. 267-281.

Andersen, T. G. (in press), "Return Volatility and Trading Volume: An Information Flow Interpretation of Stochastic Volatility," Journal of Fi $\rightarrow$ nance, 51.

Andersen, T. G., and Bollerslev, T. (in press), "Intraday Seasonality and Volatility Persistence in Financial Markets," Journal of Empirical Fi $\rightarrow$ nance, 1996.

$\rightarrow$ Baillie, R. T., and Bollerslev, T. (1989), "The Message in Daily Exchange Rates: A Conditional Variance Tale," Journal of Business \& Economic Statistics, 7, 297-305.

$\rightarrow-(1991)$, "Intra Day and Inter Market Volatility in Foreign Exchange Rates," Review of Economic Studies, 58, 565-585.

Baillie, R. T., Bollerslev, T., and Mikkelsen, H. O. (in press), "Fractional
Integrated Generalized Autoregressive Conditional Heteroskedasticity," Journal of Econometrics, 1996.

Bell, W. R., and Hillmer, S. (1984), "Issues Involved With the Seasonal Adjustment of Economic Time Series," Journal of Business \& Economic Statistics, 2, 526-534.

Bera, A. K., and Higgins, M. L. (1993), “ARCH Models: Properties, Estimation and Testing," Journal of Economic Surveys, 7, 305-366.

$\rightarrow$ Bollerslev, T. (1986), "Generalized Autoregressive Conditional Heteroskedasticity," Journal of Econometrics, 31, 307-327.

Bollerslev, T., Chou, R. Y., and Kroner, K. F. (1992), "ARCH Modelling in Finance: A Review of Theory and Empirical Evidence," Journal of Econometrics, 52, 5-59.

Bollerslev, T., and Domowitz, I. (1993), "Trading Patterns and Prices in the Interbank Foreign Exchange Market," Journal of Finance, 48, 14211443.

Bollerslev, T., and Engle, R. F. (1993), "Common Persistence in Conditional Variance," Econometrica, 61, 166-187.

Bollerslev, T., Engle, R. F., and Nelson, D. B. (1994), "ARCH Models," in Handbook of Econometrics (Vol. 4), eds. R. F. Engle and D. McFadden, Amsterdam: Elsevier Science/North-Holland, pp. 2959-3038.

Bollerslev, T., and Hodrick, R. J. (in press), "Financial Market Efficiency Tests," in Handbook of Applied Econometrics, eds. M. H. Pesaran and M. Wickens, Oxford, U.K.: Basil Blackwell.

Bollerslev, T., and Mikkelsen, H. O. (in press), "Modeling and Pricing Long-Memory in Stock Market Volatility," Journal of Econometrics, 1996.

Bollerslev, T., and Wooldridge, J. M. (1992), "Quasi Maximum Likelihood Estimation and Inference in Dynamic Models With Time Varying Covariances," Econometric Reviews, 11, 143-172.

Chan, K., Chan, K. C., and Karolyi, G. A. (1991), "Intraday Volatility in the Stock Index and Stock Index Futures Market," Review of Financial Studies, 4, 657-684.

Clark, P. (1973), "A Subordinated Stochastic Process Model With Finite Variance for Speculative Prices," Econometrica, 41, 135-156.

Dacorogna, M. M., Müller, U. A., Nagler, R. J., Olsen, R. B., and Pictet, O. V. (1993), "A Geographical Model for the Daily and Weekly Seasonal Volatility in the Foreign Exchange Market," Journal of International Money and Finance, 12, 413-438.

Diebold, F. X. (1988), Empirical Modeling of Exchange Rate Dynamics, New York: Springer-Verlag.

Drost, F. C., and Nijman, T. E. (1992), "Temporal Aggregation of GARCH Processes," unpublished manuscript, Tilburg University, Dept. of Econometrics.

$\rightarrow-$ (1993), "Temporal Aggregation of GARCH Processes," Econometrica, 61, 909-928.

Drost, F. C., and Werker, B. J. M. (in press), "Closing the GARCH Gap: Continuous Time GARCH Modelling," Journal of Econometrics, 1996.

Engle, R. F. (1982), "Autoregressive Conditional Heteroskedasticity With Estimates of the Variance of U.K. Inflation," Econometrica, 50, 9871008.

$\rightarrow$ Engle, R. F., and Bollerslev, T. (1986), "Modelling the Persistence of Conditional Variances," Econometric Reviews, 5, 1-50, 81-87.

$\rightarrow$ Engle, R. F., Ito, T., and Lin, W.-L. (1990), "Meteor Showers or Heat Waves? Heteroskedastic Intra-Daily Volatility in the Foreign Exchange Market," Econometrica, 58, 525-542.

Engle, R. F., and Ng, V. (1993), "Measuring and Testing the Impact of News on Volatility," Journal of Finance, 48, 1749-1778.

Foster, F. D., and Viswanathan, S. (1995), "Can Speculative Trading Explain the Volume-Volatility Relation?" Journal of Business \& Economic Statistics, 13, 379-396.

French, K. R., and Roll, R. (1986), "Stock Return Variances: The Arrival of Information and the Reaction of Traders," Journal of Financial Economics, 17, 5-26.

Gallant, A. R., Rossi, P. E., and Tauchen, G. (1992), "Stock Prices and Volume," Review of Financial Studies, 5, 199-242.

Ghysels, E. (1994), "On the Economics and Econometrics of Seasonality," in Advances in Econometrics, Sixth World Congress, ed. C. A. Sims, Cambridge, U.K.: Cambridge University Press, pp. 257-316.

Ghysels, E., Gouriéroux, C., and Jasiak, J. (1995), "Market Time and Asset Price Movements: Theory and Estimation,' unpublished manuscript, Université de Montréal, C.R.D.E. 
Ghysels, E., and Hall, A. (1992), "Testing for Periodicity in Some Lin $\rightarrow$ ear Macroeconomic Models," unpublished manuscript, Université de Montréal, C.R.D.E.

Ghysels, E., and Jasiak, J. (1994), "Stochastic Volatility and Time Deformation: An Application to Trading Volume and Leverage Effects," unpublished manuscript, Université de Montréal, C.R.D.E.

Gladyshev, E. G. (1961), "Periodically Correlated Random Sequences," Soviet Mathematics, 2, 385-388.

$\rightarrow$ Glosten, L. R., Jagannathan, R., and Runkle, D. E. (1993), “On the Relation Between the Expected Value and the Volatility of the Nominal Excess Return on Stocks," Journal of Finance, 48, 1779-1801.

Goodhart, C. A. E., Hall, S. G., Henry, S. G. B., and Pesaran, B. (1993) "News Effects in a High-Frequency Model of the Sterling-Dollar Exchange Rate," Journal of Applied Econometrics, 8, 1-13.

$\rightarrow$ Hamao, Y., Masulis, R. W., and Ng, V. (1990), "Correlations in Price Changes and Volatility Across International Stock Markets," Review of Financial Studies, 3, 281-307.

Hansen, L. P., and Sargent, T. J. (1990), "Recursive Linear Models of Dynamic Economics," unpublished manuscript, Stanford University, Hoover Inst.

$\rightarrow$ Harvey, C. R., and Huang, R. D. (1991), "Volatility in the Foreign Currency Futures Market," Review of Financial Studies, 4, 543-569.

Hsieh, D. A. (1989), "Modeling Heteroscedasticity in Daily ForeignExchange Rates," Journal of Business \& Economic Statistics, 7, 307317.

Hylleberg, S. A. (1986), Seasonality in Regression, New York: Academic Press.

Laux, P., and Ng, L. K. (1993), "The Sources of GARCH: Empirical Evidence From an Intraday Returns Model Incorporating Systematic anc Unique Risks," Journal of International Money and Finance, 12, 543560.

$\rightarrow$ Lee, S. W., and Hansen, B. E. (1994), "Asymptotic Theory for the GARCH $(1,1)$ Quasi-Maximum Likelihood Estimator," Econometri Theory, 10, 29-52.

$\rightarrow$ Ljung, G. M., and Box, G. E. P. (1978), "On a Measure of Lack of Fit i1 $\rightarrow$ Time Series Models," Biometrika, 65, 297-303.

Locke, P. R., and Sayers, C. L. (1993), "Intra-day Futures Price Volatility: Information Effects and Variance Persistence," Journal of Applied Econometrics, 8, 15-30.

Lopez, J. A. (1994), "Evaluating the Predictive Accuracy of Volatilit $\rightarrow$ Models," unpublished manuscript, University of Pennsylvania, Dept. of Economics.

Lumsdaine, R. L. (in press), "Consistency and Asymptotic Normality of the Quasi-Maximum Likelihood Estimator in IGARCH $(1,1)$ and $\mathrm{Co} \rightarrow$ variance Stationary IGARCH $(1,1)$ Models," Econometrica, 1996.

$\rightarrow-$ (1995), "Finite Sample Properties of the Maximum Likelihood Estimator in $\operatorname{GARCH}(1,1)$ and $\operatorname{IGARCH}(1,1)$ Models: A Monte Carl Investigation," Journal of Business \& Economic Statistics, 13, 1-10.
McCurdy, T., and Morgan, I. (1988), "Testing the Martingale Hypothesis in Deutschemark Futures With Models Specifying the Form of Heteroskedasticity," Journal of Applied Econometrics, 3, 187-202.

Müller, U. A., Dacorogna, M. M., Olsen, R. B., Pictet, O. V., Schwarz, M., and Morgenegg, C. (1990), "Statistical Study of Foreign Exchange Rates, Empirical Evidence of a Price Change Scaling Law, and Intraday Analysis," Journal of Banking and Finance, 14, 1189-1208.

$\rightarrow$ Nelson, D. B. (1990), "ARCH Models as Diffusion Approximations," Journal of Econometrics, 45, 7-38.

_ (1991), "Conditional Heteroskedasticity in Asset Returns: A New Approach," Econometrica, 59, 347-370.

Nelson, D. B., and Cao, C. Q. (1992), "Inequality Constraints in the Univariate GARCH Model," Journal of Business \& Economic Statistics, 10, 229-235.

Nerlove, M., Grether, D. M., and Carvalho, J. L. (1979), Analysis of Economic Time Series: A Synthesis, New York: Academic Press.

Nijman, T., and Sentana, E. (in press), "Marginalization and Contemporaneous Aggregation in Multivariate GARCH Processes," Journal of Econometrics, 1996.

$\rightarrow$ Osborn, D. R. (1988), "Seasonality and Habit Persistence in a Life-Cycle Model of Consumption," Journal of Applied Econometrics, 3, 255-266.

(1991), "The Implications of Periodically Varying Coefficients for Seasonal Time Series Processes,” Journal of Econometrics, 48, 373-384.

Osborn, D. R., and Smith, J. P. (1989), "The Performance of Periodic Autoregressive Models in Forecasting Seasonal U.K. Consumption," Journal of Business \& Economic Statistics, 7, 117-127.

Pagan, A. R., and Schwert, G. W. (1990), "Alternative Models for Conditional Stock Volatility," Journal of Econometrics, 45, 267-290.

Schwarz, G. (1978), "Estimating the Dimension of a Model," The Annals of Statistics, 6, 461-464.

$\rightarrow$ Schwert, G. W. (1990), "Indexes of U.S. Stock Prices from 1802 to 1987," Journal of Business, 63, 399-426.

Tauchen, G. E., and Pitts, M. (1983), "The Price Variability-Volume Relationship on Speculative Markets," Econometrica, 51, 485-505.

Taylor, S. (1994), "Modelling Stochastic Volatility," Mathematical Finance, 4, 183-204.

Tiao, G. C., and Grupe, M. R. (1980), "Hidden Periodic Autoregressive Moving Average Models in Time Series Data," Biometrika, 67, 365373.

Weiss, A. A. (1986), “Asymptotic Theory for ARCH Models: Estimation and Testing," Econometric Theory, 2, 107-131.

West, K. D., and Cho, D. (1995), "The Predictive Ability of Several Models of Exchange Rate Volatility," Journal of Econometrics, 69, 367-391.

West, K. D., Edison, H. J., and Cho, D. (1993), "A Utility Based Comparison of Some Models of Exchange Rate Volatility," Journal of International Economics, 35, 23-45.

Wood, R., McInish, T., and Ord, J. K. (1985), “An Investigation of Transaction Data for NYSE Stocks," Journal of Finance, 40, 723-739. 